\title{
Single-Layer Based Algorithms for Solving the Inverse Problem of ECG
}

\author{
Danila Potyagaylo $^{1}$, Mikhail Chmelevsky ${ }^{1,2}$, Alexander Kalinin ${ }^{1}$ \\ ${ }^{1}$ EP Solutions SA, Yverdon-les-Bains, Switzerland \\ ${ }^{2}$ Almazov National Medical Research Centre, Saint Petersburg, Russia
}

\begin{abstract}
Recently, the equivalent single layer (ESL) source model, along with the respective regularization matrix, was introduced for solving the ECG imaging (ECGI) problem with the boundary element method (BEM).

In the present work, we extend the proposed formulation by three additional ECGI methods. The first algorithm is based on substitution of the pseudoinverse transfer matrix for the single layer into the alternative BEM equation (ESL-EP). The second extension consists in an iterative update of the two BEM equations, while alternating between the epicardial potentials and their normal derivative. The third solution approach results from weighting the initial ESL solution with ESL-EP. We tested the proposed algorithms on three in silico and twenty clinical cases for ectopic ventricular excitation and quantitatively compared their performance in terms of reconstructed activation times and earliest activation areas, respectively.

The methods featured distinct results with the best mean localization accuracy obtained by the initial ESL and the weighted approaches. ESL-EP and the iterative algorithm facilitated correct classification of the excitation onset being endocardial.
\end{abstract}

\section{Introduction}

ECG imaging (ECGI) is a modality combining torso geometrical information with body surface potential maps (BSPMs) in order to non-invasively compute electrical cardiac sources. The underlying mathematical problem is illposed, which is caused by the human body attenuating and blurring the electrical currents leaving the heart. One of the consequences of this ill-posedness is solution's ambiguity making reconstruction of endocardial sources extremely challenging. With this respect, the equivalent single layer (ESL) source model was recently introduced and shown to improve ECGI accuracy on the endocardium using three simulated cases of ectopic ventricular excitation [1].

In the present work, we propose two alternative numerical formulations of the ESL-based inverse problem and introduce a combined approach, resulting in four distinct
ECGI algorithms. Furthermore, we quantitatively assess performance of these approaches for the in silico data used in [1] and clinical pacing data from [2].

\section{Methods}

\subsection{Inverse reconstructions}

For the inverse modeling, we applied the boundary element method (BEM) under the assumption of the body volume conductor being homogeneous and constrained by triangulated torso and cardiac surfaces, $\Gamma_{0}$ and $\Gamma_{1}$ respectively.

\subsubsection{Epicardial Potential (EP)}

The classical BEM approach to the forward ECG problem results in the following system of linear equations connecting electrical potentials $u_{l}$ and their normal derivatives $q_{l}$ on the surfaces $\Gamma_{l}(l=0,1)[1,3]$ :

$$
\begin{aligned}
& H_{00} u_{0}+H_{01} u_{1}=G_{01} q_{1} \\
& H_{10} u_{0}+H_{11} u_{1}=G_{11} q_{1}
\end{aligned}
$$

Due to insulating properties of the body, $q_{0}$ is equal 0 and, thus, excluded from the above equations. With a few algebraic manipulations, one can arrive at the relation between the heart surface potentials $u_{1}$ and body surface electrocardiograms $u_{0}$ :

$$
\left(-H_{01}+G_{01} G_{11}^{-1} H_{11}\right) u_{1}=\left(H_{00}-G_{01} G_{11}^{-1} H_{10}\right) u_{0}
$$

or

$$
A u_{1}=f_{0}
$$

in short.

\subsubsection{Equivalent Single Layer (ESL) Poten- tial}

As demonstrated in [1], after defining the equivalent single layer density function $w_{1}$ as follows:

$$
u_{1}=G_{11} w_{1}
$$


we can formulate the corresponding inverse problem of ECG in terms of $w_{1}$ :

$$
4 \pi G_{01} w_{1}=f_{0}
$$

with the right-hand side being equal to those in (3). The function $w_{1}$ was shown to possess a have a higher level of spatio-temporal regularity compared to $u_{1}$ [1]. Furthermore, splitting the transfer matrix $A$ from (3) into the product of an ill-conditioned matrix $G_{01}$ and a wellconditioned $G_{11}^{-1}$ exhibited superior accuracy results on the endocardial heart surface for in silico focal activation patterns.

\subsubsection{ESL-EP}

We can define a pseudoinverse of the matrix $G_{01}$ to solve the ESL problem (5):

$$
G_{01_{\lambda}}^{-1}=\left(G_{01}^{T} G_{01}+\lambda I\right)^{-1} G_{01}^{T}
$$

with $I$ being an identity matrix and $\lambda$ the regularization parameter restricting the ESL norm in (5).

Substituting this pseudoinverse approximation into the original BEM statement (1), we obtain the following system:

$$
\begin{aligned}
G_{01_{\lambda}}^{-1}\left(H_{00} u_{0}+H_{01} u_{1}\right) & =q_{1} \\
G_{11}^{-1}\left(H_{10} u_{0}+H_{11} u_{1}\right) & =q_{1}
\end{aligned}
$$

implying

$$
G_{01_{\lambda}}^{-1}\left(H_{00} u_{0}+H_{01} u_{1}\right)=G_{11}^{-1}\left(H_{10} u_{0}+H_{11} u_{1}\right)
$$

After rearranging the terms for $u_{0}$ and $u_{1}$ and denoting

$$
\begin{aligned}
& T_{u_{1}}=G_{01_{\lambda}}^{-1} H_{01}-G_{11}^{-1} H_{11} \\
& T_{u_{0}}=G_{11}^{-1} H_{10}-G_{01_{\lambda}}^{-1} H_{00}
\end{aligned}
$$

we invert the well-posed matrix $T_{u_{1}}$ to find

$$
u_{1}=T_{u_{1}}^{-1} T_{u_{0}} u_{0}
$$

This solution scheme regularizes the problem in the ESL space and, subsequently, makes use of both BEM equations (1) by eliminating the normal derivative $q_{1}$ and expressing $u_{1}$ through $u_{0}$. It is worth noting that the matrix $T_{u_{1}}$ is well-conditioned, meaning that regularization occurs only at the step of $G_{01}$ inversion.

\subsubsection{Iterative ESL}

Alternatively, we can employ the pseudoinverse formulation for $G_{01}$ (2.1.3) and substitute it into the BEM equations (1) to express both unknown variables $u_{1}$ and $q_{1}$ :

$$
\begin{aligned}
& u_{1}=H_{11}^{-1}\left(G_{11} q_{1}+H_{10} u_{0}\right) \\
& q_{1}=G_{01_{\lambda}}^{-1}\left(H_{00} u_{0}+H_{01} u_{1}\right)
\end{aligned}
$$

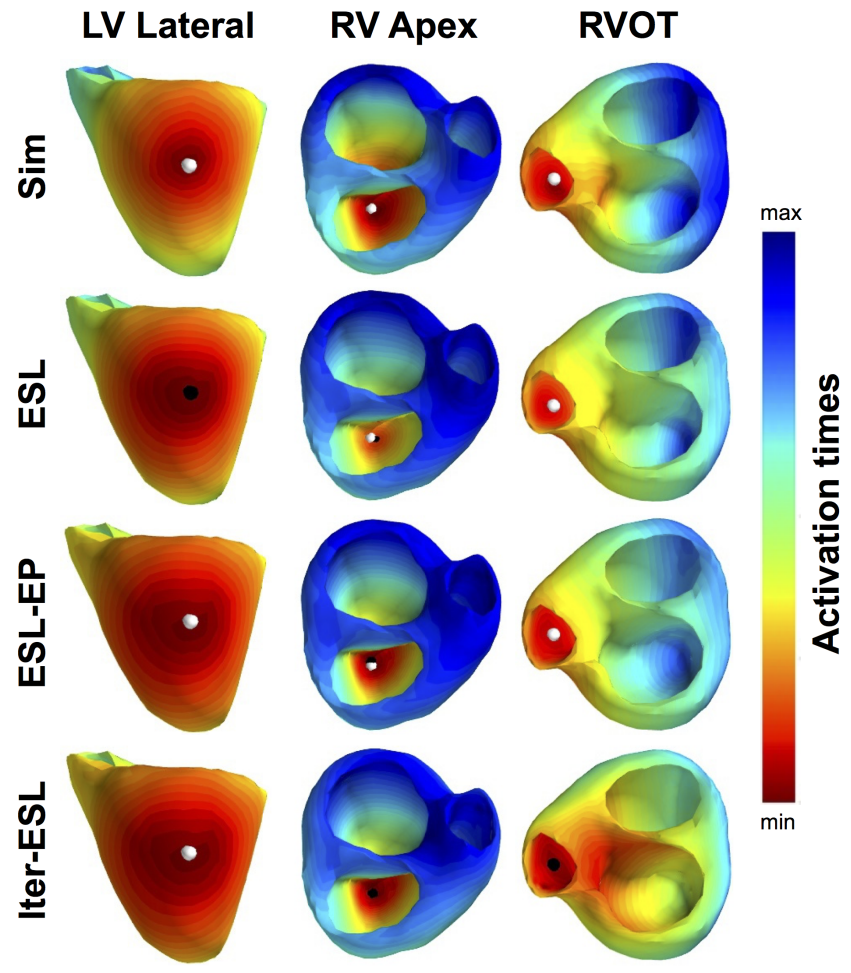

Figure 1: Activation times for simulated data. White dots represent the true simulated excitation origin, the black ones mark the ECGI calculated activation onset.

Then we iterate over the equations (10a) and (10b) to obtain a regularized solution. Starting with an initial estimate $\tilde{q}_{1}=0$ we update $\tilde{u}_{1}$ and $\tilde{q}_{1}$ by (10a) and (10b), respectively. The iterations can be performed either until the relative error between measured and approximated body surface potential maps $u_{0}$ and $\tilde{u}_{0}$ becomes smaller than a predefined $\epsilon$ parameter or until the maximum number of iterations is reached. In the present work, we ran four iterations before terminating the loop.

\subsubsection{ESL Solution Stack}

Having multiple solutions of the same problem at hand, we can, using machine-learning vocabulary, stack them with the hope that a new combination would possess desirable features from all components. In the present work we weighted solutions of the ESL and ESL-EP based problems (4) and (9) with coefficients of 0.6 and 0.4 , respectively.

\subsection{Activation Times Calculation}

For computing activation times (ATs) from the resulting electrograms (EGs), we used the activation direction method described in [4]. In short, we first find the gra- 
dient field of electrical potentials on the heart surface and then its projection on the tangential plane $\mathbf{g}\left(\mathbf{x}, \mathbf{t}_{\mathbf{k}}\right)$. Afterwards, the time instances corresponding to the maximum gradient's amplitude at all cardiac nodes and the respective gradient's values are extracted:

$$
\begin{aligned}
t_{k_{1}} & =\underset{t_{k}}{\arg \max }\left\|\mathbf{g}\left(\mathbf{x}, \mathbf{t}_{\mathbf{k}}\right)\right\| \\
X & =\mathbf{g}\left(\mathbf{x}, \mathbf{t}_{\mathbf{k}_{\mathbf{1}}}\right)
\end{aligned}
$$

From the known function $X$, we approximate the activation times with the scalar function $a(\mathbf{x})$ minimizing the following functional:

$$
I=\int_{\Gamma_{1}}|\nabla a-X|
$$

\subsection{Simulation Setup}

To exemplary compare performance of the ESL, ESLEP and iterative ESL methods, we used three simulations of ventricular focal excitation starting from the epicardial left ventricular (LV) lateral wall, right ventricular outflow tract (RVOT) and right ventricular (RV) endocardial apex. For further description of the simulation setup, the reader is referred to [1]. After contaminating the simulated BSPMs with $20 \mathrm{~dB}$ of white Gaussian noise, inversely calculating EGs and deriving the activation times, we assessed the results in terms of ATs correlation and localization accuracy for the earliest excitation site.

\subsection{Clinical Data}

The same patient data as reported in [2] were used to evaluate the accuracy of the proposed ECGI methods. In a nutshell, ten patients with previously implanted CRT devices were subject to right and left ventricular isolated pacings, resulting in total of twenty analyzed BSPMs. The pacemaker leads locations were exactly known from the CT scans, allowing for quantitative evaluation of excitation origin's localization. As all LV pacings had epicardial and RV pacings endocardial origin we also computed the corresponding classification rate for the introduced ECGI methods.

\section{Results}

\subsection{ECGI for Simulation Data}

Activation times for the simulations and ESL, ESL-EP and Iterative ESL (Iter-ESL) reconstructions are shown in Fig. 1. For the LV lateral case, the ESL based origin coincided with the simulated one, whereas ESL-EP and iterative ESL detected the earliest excitation site on the endocardium with localization error (LE) of $9 \mathrm{~mm}$. For RV apex and RVOT cases, the iterative ESL method resulted in the perfect reconstruction. ESL for the RV apex case, and ESL and ESL-EP for the RVOT case wrongly estimated the activation onset to belong to the epicardial surface. The LEs for the RV apex case for ESL and ESL-EP were 4 and $3 \mathrm{~mm}$, respectively. For the RVOT simulation, these methods resulted in the same error of $5 \mathrm{~mm}$.

\subsection{ECGI for Clinical Pacing Data}

The box plots for Euclidean LEs between the pacemaker leads and ECGI based excitation origins are provided in Fig. 2. The errors (mean \pm standard deviation) for ESL, ESL-EP, Iter-ESL and ESL-Stack were $17 \pm 10,19 \pm 15$, $22 \pm 20$ and $16 \pm 12 \mathrm{~mm}$, respectively. Interestingly, all methods shared the same outlier. For this LV pacing, in Fig. 3 we show the resulting activation maps together with the reconstructed EGs at the true pacing position and the found one. The classification rate of correctly detecting epicardial and endocardial origins for LV and RV pacings, respectively, are given by Table 1 . Only the ESL method resulted in a mixed classification rates, while other methods were able to correctly predict all endocardial origins and none of the LV epicardial ones.

\section{Discussion}

The obtained LEs for the considered simulation cases were comparable among all introduced methods. However, the resulting activation maps already indicated algorithms' differences that were confirmed by analysis of the clinical data. The lowest mean LEs were observed for the ESL and ESL-Stack methods, though there was no significant difference between all approaches $(p>0.05)$. Remarkably, ESL-EP and Iter-ESL approaches were able to correctly classify all RV pacings to be endocardial and none of the LV ones to originate from the epicardium. In contrast, the ESL method resulted in a mixed correct rate, which was, therefore, improved for the RV and deteriorated for the LV cases when combined with the ESLEP. These observations suggest some numerical properties of the involved matrices favouring endocardial sources. Compared to the LEs reported in [2], the ESL-based approaches combined with ADM resulted in worse accuracy for both LV and RV but higher correction rate for the considered RV pacings, suggesting that both model-based and data-driven methodologies for ECGI should be pursued in parallel.

Apart from the EG reconstruction, a technique for computing activation times play also a crucial role in the quality of final ECGI results and their interpretability. We visually examined the temporal evolution of the potential distribution on the cardiac surface for the clinical outlier case and discovered a distinct excitation onset within $1 \mathrm{~cm}$ of 


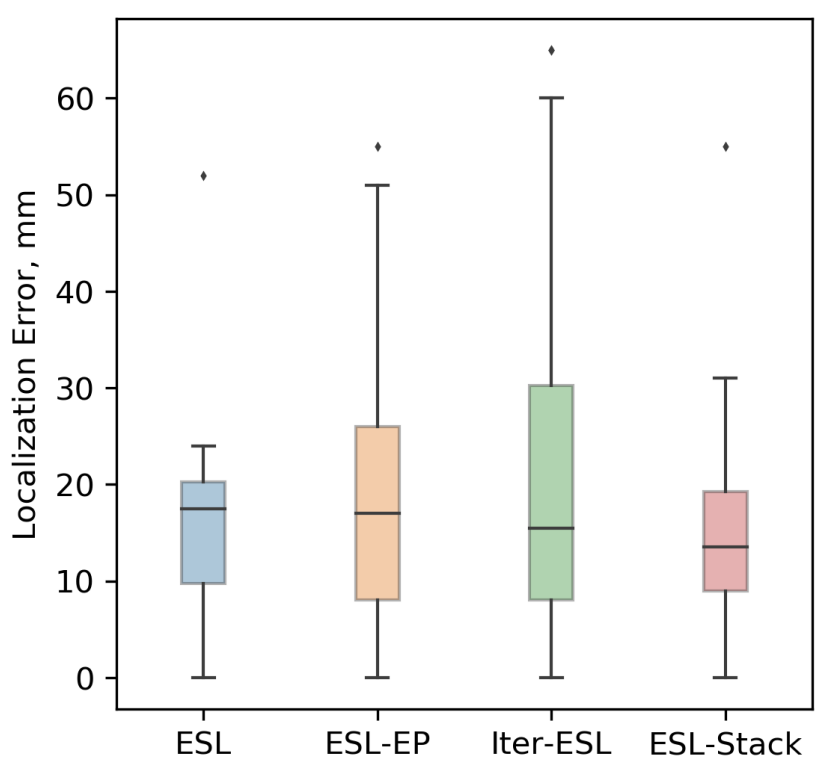

Figure 2: LEs for clinical pacing data. The box plot's bar refers to the median. Whiskers indicate 1.5 IQR past the lower $(75 \%)$ and higher $(25 \%)$ quartiles.

Table 1: Endo-/epicardial classification rate for clinical pacing data.

\begin{tabular}{|c|c|c|c|c|}
\hline & ESL & ESL-EP & Iter-ESL & ESL-Stack \\
\hline LV & 0.8 & 0 & 0 & 0 \\
\hline RV & 0.4 & 1 & 1 & 1 \\
\hline
\end{tabular}

the true origin. Possibly, complex wave propagation due to some slow conducting areas in the LV affected activation maps computation worsening the final result. The used ADM method is based on the heat flow approximation over a smooth manifold due to the pre-calculated gradient field. This approach seems to oversmooth the activation propagation, thus needing a more careful scheme for weighting spatio-temporal information contained in the EGs.

\section{Conclusions}

The present work introduced two novel numerical approaches for solving the ESL-based ECGI problem. The promising results obtained on both simulation and clinical data indicate a high potential of the proposed methods for imaging endocardial sources. For enhanced clinical usability of any ECGI tool, post-processing methods, in general, and activation times calculation, in particular, should properly weight the spatio-temporal EG's components in order to avoid oversmoothing or frequently seen patchy patterns in the resulting ATs. Finally, we showed that combination of EGs inversely computed with distinct methods might
ESL

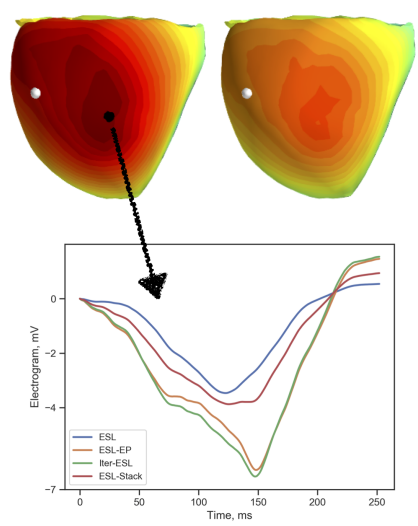

Iter-ESL ESL-Stack

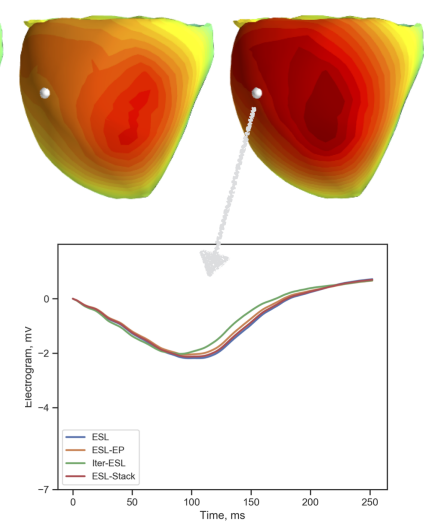

Figure 3: In the upper row the ADM based activation maps are visualized for the $L V$ case featuring the highest $L E$. For ESL-EP, Iter-ESL and ESL-Stack, the found origin was located on the endocardium. The lower row depicts the inversely reconstructed EGs at the found positions (left) and the cardiac node closest to the true pacemaker lead.

improve an overall ECGI performance.

\section{References}

[1] Kalinin A, Potyagaylo D, Kalinin V. Solving the inverse problem of electrocardiography on the endocardium using a single layer source. Frontiers Physiol 2019;10:58.

[2] Potyagaylo D, Chmelevsky M, Van Dam P, Budanova M, Zubarev S, Treshkur T, Lebedev D. Ecg adapted fastest route algorithm to localize the ectopic excitation origin in crt patients. Front Physiol 2019;10.

[3] Gulrajani RM. The forward and inverse problems of electrocardiography. IEEE Eng Med Biol 1998;17(5):84-101.

[4] Denisov A, Zakharov E, Kalinin A. Method for determining the projection of an arrhythmogenic focus on the heart surface, based on solving the inverse electrocardiography problem. Math Models and Comput Simul 2012;4(6):535-540.

Address for correspondence:

Danila Potyagaylo

EP Solutions SA,

Avenue des Sciences 13, 1400 Yverdon-les-Bains, Switzerland danila.potyagaylo@ep-solutions.ch 